\title{
ON JORDAN ALGEBRAS AND SOME UNIFICATION RESULTS
}

\author{
Florin F. Nichita \\ Simion Stoilow Institute of Mathematics of the Romanian Academy \\ 21 Calea Grivitei, 010702 Bucharest, Romania
}

\begin{abstract}
This paper is based on a talk given at the 14-th International Workshop on Differential Geometry and Its Applications, hosted by the Petroleum Gas University from Ploiesti, between July 9-th and July 11-th, 2019. After presenting some historical facts, we will consider some geometry problems related to unification approaches. Jordan algebras and Lie algebras are the main non-associative structures. Attempts to unify non-associative algebras and associative algebras led to UJLA structures. Another algebraic structure which unifies non-associative algebras and associative algebras is the Yang-Baxter equation. We will review topics relared to the Yang-Baxter equation and Yang-Baxter systems, with the goal to unify constructions from Differential Geometry.
\end{abstract}

Keywords: Jordan algebras, Lie algebras, associative algebras, Yang-Baxter equations

MSC-class: 16T05, 16T25, 53C05, 51N20, 01A60, 00A05 (Primary), 17C05, 17C50 (Secondary)

\section{INTRODUCTION}

This paper is a survey paper, but it also presents new results. It emerged after our lectures given at the 9-th Congress of Romanian Mathematicians (CRM 9, Galati, Romania, June-July 2019) and 14-th International Workshop on Differential Geometry and Its Applications (DGA 14, UPG Ploiesti, July 2019).

We begin with some historical remarks. Vrănceanu proposed the study of spaces with constant affine connection associated to finite-dimensional real Jordan algebras in 1966. This study was afterwards developed by Iordanescu, Popovici and Turtoi (see the Comments on page 31 from [14]). Dan Barbilian proved that the rings which can be the underlying rings for projective geometries are (with a few exceptions) rings with a unit element in which any one-sided inverse is a two-sided inverse (see [14]). Another big Romanian geometer was G. Tzitzeica, and some of his contributions to mathematics will be presented in our next section.

A substantial part of this paper is dedicated to unifying structures. The apparition of the Yang-Baxter equation ([36]) in theoretical physics and statistical mechanics (see $[44,2,3])$ has led to many applications in these fields and in quantum groups, quantum computing, knot theory, braided categories, analysis of integrable systems, quantum mechanics, etc (see [34]). The interest in this equation is growing, as new properties of it are found, and its solutions are not classified yet. This equation can be understood as an unifying equation (see, for example, $[35,20,21,15]$ ). Another unification of 
non-associative structures, was obtained using the UJLA structures ([15, 16]), which could be seen as structures which comprise the information encapsulated in associative algebras, Lie algebras and Jordan algebras.

We now refer to important results obtained under the guidance of St. Papadima. Some presentations at DGA 14 were dedicated to his memory (see $[4,38,28,41]$ ). It was observed during DGA 14, that some results from [38] can be extended for the virtual braid groups from [4]. Also, the techniques of [41] can be considered in the framework of UJLA structures. The Yang-Baxter equation plays an important role in knot theory. Dăscălescu and Nichita have shown in [8] how to associate a Yang-Baxter operator to any algebra structure over a vector space, using the associativity of the multiplication. Turaev has described in [43] a general scheme to derive an invariant of oriented links from a Yang-Baxter operator, provided this one can be "enhanced". The invariant which was obtained from those Yang-Baxter operators is the Alexander polynomial of knots. Thus, in a way, the Alexander polynomial is the knot invariant corresponding to the axioms of (unitary associative) algebras.

The current paper is organised as follows. The next section deals with TzitzeicaJohnson's theorem. In Sections 3 and 4 we present the Yang-Baxter equations and the Yang-Baxter systems (including the set-theoretical Yang-Baxter equation). These will be followed by a section on Jordan algebras, UJLA structures and unification constructions in Differential Geometry. A conclusion section will end this article.

\section{On TZITZEicA-Johnson's THEOREM AND PICTORIAL MATHEMATICS}

In his talk at DGA 12, Florin Caragiu explained that there exists a special mathematical discourse, called "proofs without words", which uses pictures or diagrams in order to boost the intuition of the reader (see [7]). Pictorial (diagrammatic) style of mathematical language is much appreciated by both educators and researchers in mathematics (see, also, [26]). Very easy to be grasped, some pictorial style problems need an entirely "artillery" in order to be cracked.

We now consider the Tzitzeica-Johnson's problem (see, for example, [26]). We start with three circles of the same radius $r$. The intersection points of pairs of circles are denoted by A, B, C and the common point of intersection of the three circles is denoted by $\mathrm{O}$ (see the Figure 1 below).

Figure 1. The three coins problem (Tzitzeica - 1908, Johnson 1916). Under the above assumptions, there exists a circle with radius $r$, passing through the points $\mathrm{A}, \mathrm{B}$ and $\mathrm{C}$.

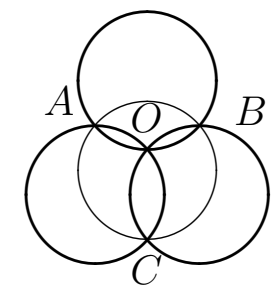

We now propose a new construction (in the Figure 2). 
Figure 2. The tangent to the third circle in $\mathrm{O}$ meets the line $\mathrm{BC}$ in $\mathrm{R}$.

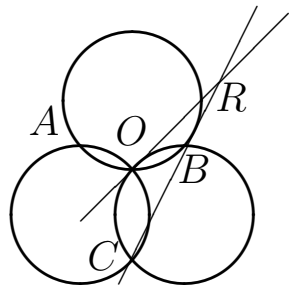

At this moment, we can present our Theorem: under the above assumptions, the tangents to the circles in $\mathrm{O}$ meet the lines $\mathrm{AB}, \mathrm{AC}$ and $\mathrm{BC}$ in three colinear points (see the Figure 3 below).

Figure 3. Theorem. The points $\mathrm{P}, \mathrm{Q}$ and $\mathrm{R}$ are colinear.

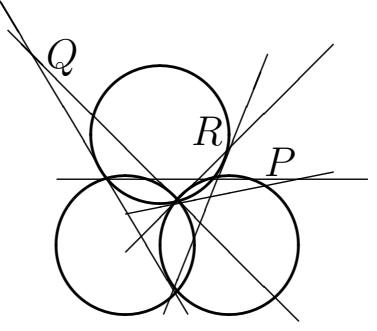

The proof of the above theorem is based on the properties of the power of a circle and on the Desargues' theorem. It is the limit case of the following picture.

FiguRE 4 . The limit case of the following picture implies our theorem.

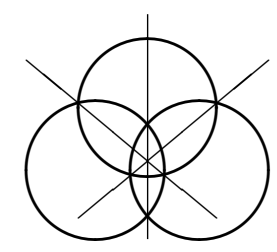

The above problems can be included in a more general scheme (see the Figure 5).

Figure 5. A mathematical scheme. If $\mathcal{X}, \mathcal{Y}$ and $\mathcal{Z}$ have certain properties, then there exists a related $\mathcal{T}$, with the same properties.

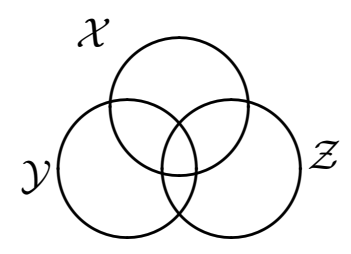


The dual of the Desargues' Theorem can be interpretated in the light of the above scheme by using arrangements of (three) colored lines.

The hypothesis:

$\mathcal{X}=\{(d, f, h)$ such that $d \cap f \cap h=P\}$

$\mathcal{Y}=\left\{\left(d^{\prime}, f, h^{\prime}\right)\right.$ such that $\left.d^{\prime} \cap f \cap h^{\prime}=Q\right\}$

$\mathcal{Z}=\left\{\left(d^{\prime \prime}, f, h^{\prime \prime}\right)\right.$ such that $\left.d^{\prime \prime} \cap f \cap h^{\prime \prime}=R\right\}$

$\mathcal{X} \cap \mathcal{Y} \cap \mathcal{Z}=\{f\}$

$\mathcal{X} \cap \mathcal{Y}=\left\{A, A^{\prime}\right\} \cup\{f\}$

$\mathcal{Y} \cap \mathcal{Z}=\left\{B, B^{\prime}\right\} \cup\{f\}$

$\mathcal{X} \cap \mathcal{Z}=\left\{C, C^{\prime}\right\} \cup\{f\}$

Conclusion:

There exist a triple $(l, m, n)$ through $A, A^{\prime}, B, B^{\prime}, C, C^{\prime}$.

From the phylosophical point of view, one could include in this scheme the inclussionexclusion principle:

$|X \cup Y \cup Z|=|X|+|Y|+|Z|-|X \cap Y|-|X \cap Z|-|Y \cap Z|+|X \cap Y \cap Z|$.

Let us explain this analogy. Once we have information about the cardinality of $X, Y, Z, X \cap Y, X \cap Z, Y \cap Z$ and $X \cap Y \cap Z$, then there exists a $T$, related to $X, Y$ and $Z$, whose cardinality can be also computed. Of course, in this case $T=X \cup Y \cup Z$.

In order to illustrate better this analogy, let us suppose further that $|X|=|Y|=$ $|Z|=7,|X \cap Y|=|X \cap Z|=|Y \cap Z|=1$ and $|X \cap Y \cap Z|=1$. Then, there exists a set $T$, with $|T|=7$ which contains elements from all possible (sub)sets.

Notice that Tzitzeica-Johnson's Theorem has many generalizations ([26]) and interpretations related to the above mathematical scheme (from Figure 5).

Thus, Tzitzeica-Johnson's Theorem can be interpretated in terms of disks:

(i) "If three disks of the same radius have a common point of intersection, then they contain inside of their union a forth disk with the same radius."

(ii) "If three disks $\mathcal{X}, \mathcal{Y}$ and $\mathcal{Z}$ of the same radius have a common point, then there exists a forth disk of the same radius which includes $(\mathcal{X} \cap \mathcal{Y}) \cup(\mathcal{X} \cap \mathcal{Z}) \cup(\mathcal{Y} \cap \mathcal{Z})$."

It is an open problem to prove a similar statement for a domain bounded by an arbitary closed convex curve. In other words we conjecture that if we consider a domain $\mathcal{X}$ bounded by an arbitary closed convex curve, and two copies of $\mathcal{X}$, denoted by $\mathcal{Y}$ and $\mathcal{Z}$ such that $\mathcal{X} \cap \mathcal{Y} \cap \mathcal{Z} \neq \emptyset$, then there exists another copy of $\mathcal{X}$, denoted by $\mathcal{T}$ such that $(\mathcal{X} \cap \mathcal{Y}) \cup(\mathcal{X} \cap \mathcal{Z}) \cup(\mathcal{Y} \cap \mathcal{Z}) \subset \mathcal{T}$.

We now consider other types of geometry problems.

In his best-seller book [10], P. Greco (see [37] - there is a puzzle there!) refers to a problem which can be generalized as follows.

OPEN PROBLEMS. For an arbitrary convex closed curve, we consider the length of largest barycentric diameter $(D)$. Here, the largest barycentric diameter is the longest segment through the center of mass with the endpoints on the given curve. In a similar manner, one can define the smallest barycentric diameter (d).

i) If $L$ is the length of the given curve and the domain inside the given curve is a convex set, then we conjecture that:

$$
\frac{L}{D} \leq \pi \leq \frac{L}{d} .
$$


(ii) If $A$ is the area inside the given curve, the equation

$$
x^{2}-\frac{L}{2} x+A=0
$$

and its implications are not completely understood. For example, if the given curve is an ellipse, solving this equation in terms of the semi-axes of the ellipse is an unsolved problem.

The second part of the above conjecture is related to [1].

Another idea related to [10] and to the Euler's relation, $e^{\pi i}+1=0$, is the following relation containing $\pi:\left|e^{i}-\pi\right|>e$. It is very good approximation, and it has a pictorial interpretation (see the Figure 6).

Figure 6. An interpretation for the formula $\left|e^{i}-\pi\right| \approx e$.

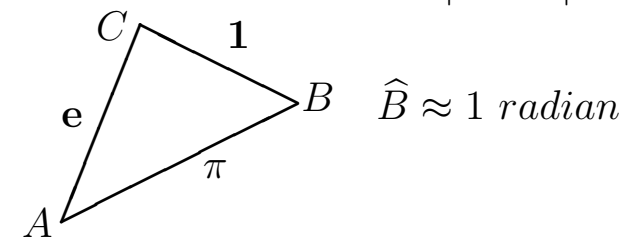

\section{YANG-BAXTER EQUATIONS}

In the next sections, we will work over a generic field $k$. The tensor products will be defined over $k$.

This section was inspired by two papers, [25, 42], and it begins with an introduction to the Yang-Baxter equation.

Let $V$ be a vector space over $k$. Let $I=I_{V}: V \rightarrow V$ be the identity map of the space $V$.

We denote by $\tau: V \otimes V \rightarrow V \otimes V$ the twist map defined by $\tau(v \otimes w)=w \otimes v$.

Now, we will give the main notations for introducing the Yang-Baxter equation.

For $R: V \otimes V \rightarrow V \otimes V$ a $k$-linear map, let $R^{12}=R \otimes I, R^{23}=I \otimes R: V \otimes V \otimes V \rightarrow$ $V \otimes V \otimes V$. In a similar manner, we denote by $R^{13}$ a linear map acting on the first and third component of $V \otimes V \otimes V$. It turns out that $R^{13}=(I \otimes \tau)(R \otimes I)(I \otimes \tau)$.

Definition 3.1. A Yang-Baxter operator is $k$-linear map $R: V \otimes V \rightarrow V \otimes V$, which satisfies the braid condition (the Yang-Baxter equation):

$$
R^{12} \circ R^{23} \circ R^{12}=R^{23} \circ R^{12} \circ R^{23} .
$$

We also require that the map $R$ is invertible.

Remark 3.2. Some examples of Yang-Baxter operators are the following: $R=\tau$ (i.e., $R(a \otimes b)=b \otimes a)$, and $R=I \otimes I \quad$ (i.e., $\quad R(a \otimes b)=a \otimes b)$. 
Remark 3.3. An important observation is that if $R$ satisfies (2) then both $R \circ \tau$ and $\tau \circ R$ satisfy the $Q Y B E$ (the quantum Yang-Baxter equation):

$$
R^{12} \circ R^{13} \circ R^{23}=R^{23} \circ R^{13} \circ R^{12} .
$$

Remark 3.4. The equations (2) and (3) are equivalent.

Remark 3.5. The following construction of Yang-Baxter operators is described in [8]. If $A$ is a $k$-algebra, then for all non-zero $r, s \in k$, the linear map

$$
R_{r, s}^{A}: A \otimes A \rightarrow A \otimes A, \quad a \otimes b \mapsto s a b \otimes 1+r 1 \otimes a b-s a \otimes b
$$

is a Yang-Baxter operator.

The inverse of $R_{r, s}^{A}$ is $\left(R_{r, s}^{A}\right)^{-1}(a \otimes b)=\frac{1}{r} a b \otimes 1+\frac{1}{s} 1 \otimes a b-\frac{1}{s} a \otimes b$.

Turaev has described in [43] a general scheme to derive an invariant of oriented links from a Yang-Baxter operator, provided this one can be "enhanced".

The Jones polynomial [18] and its two-variable extensions, namely the Homflypt polynomial [9, 39] and the Kauffman polynomial [19], can be obtained in that way by "enhancing" some Yang-Baxter operators obtained in [17]. Those solutions of the Yang-Baxter equation are associated to simple Lie algebras and their fundamental representations. The Alexander polynomial can be derived from a Yang-Baxter operator as well, using a slight modification of Turaev's construction [27].

The only invariant which can be obtained from the Yang-Baxter operators (4) is the Alexander polynomial of knots. Thus, in a way, the Alexander polynomial is the knot invariant corresponding to the axioms of (unitary associative) algebras (cf. [24]).

Note that specializations of the Homflypt polynomial had to be expected from those Yang-Baxter operators since they have degree 2 minimal polynomials.

Remark 3.6. There is a similar terminology for the set-theoretical Yang-Baxter equation. In this case $V$ is replaced by a set $X$ and the tensor product by the Cartesian product. We will explain this definition in the next examples below.

Let $X$ be a set containing three logical sentences $p, q, r$ (i.e., $p, q, r \in X$ ). We can choose $X$ as rich as we wish for the moment. Later, we will try to find the smallest $X$ which fits for our theory.

Let $R: X \times X \rightarrow X \times X$, be defined by $R(p, q)=(p \vee q, p \wedge q)$.

It follows that

$$
(R \times I) \circ(I \times R) \circ(R \times I)=(I \times R) \circ(R \times I) \circ(I \times R) .
$$

One way to check that (5) holds is to make a table with values for $p, q, r$.

Remark 3.7. Another interesting solution to the set-theoretical Yang-Baxter equation is the following.

Let $R: X \times X \rightarrow X \times X$, be defined by $R(p, q)=(p \rightarrow q, p)$.

Again, one way to check the above statement is to make a table with values for $p, q, r$.

Let us denote $R(p, q)=\left(p_{1}, q_{2}\right)$. The set-theoretical Yang-Baxter equation can be expressed as 


$$
\left(p_{101}, q_{212}, r_{020}\right)=\left(p_{010}, q_{121}, r_{212}\right)
$$

It is now the moment to discuss about $X$.

Open problems. What can be said about $X$ in general? What is the smallest $X$ for which $R$ is well-defined? One could consider a set $X$ containing more than three logical sentences. What is the interpretation of the set-theoretical Yang-Baxter solutions in this case?

We can go a step further and consider an algebra of type $(2,2),(A, *, \circ)$, and call the operations $*$ and $\circ Y B$ conjugated if $R(a, b)=(a * b, a \circ b)$ satisfies the set-theoretical Yang-Baxter equation.

We propose the study of algebras with two operations which are YB conjugated. Groups, distributive lattices, and self-distributive laws can be considered as objects with operations which are YB conjugated (compare with [5]).

Remark 3.8. We now consider other (systems of) equations for a k-linear map $R$ : $V \otimes V \rightarrow V \otimes V:$

$$
\begin{gathered}
R^{12} \circ R^{23}=R^{13} \circ R^{12}=R^{23} \circ R^{13} \\
R^{23} \circ R^{12}=R^{12} \circ R^{13} \\
(8) \\
\begin{array}{c}
(9) \\
(10)
\end{array} \\
\left(R^{12} \circ R^{23} \circ R^{12} \circ R^{23} \circ R^{12} \circ R^{23}=R^{23} \circ R^{13} \circ R^{23} \circ R^{12}\right. \\
(11) \\
(12) \\
(13)
\end{gathered}
$$

Theorem 3.9. If a $k$-map $R: V \otimes V \rightarrow V \otimes V$ verifies (7) and (8) then $R$ is a common solution for (2) and (3).

If a $k$-map $R: V \otimes V \rightarrow V \otimes V$ verifies (2) and (3) then it is a solution for (9).

If a $k$-map $R: V \otimes V \rightarrow V \otimes V$ verifies (2) or (3) then $R$ is a solution for (10).

If a $k$-map $R: V \otimes V \rightarrow V \otimes V$ verifies (2) or (3) then it is a solution for (11).

Proof. We only prove the first claim: $R^{23} \circ R^{12} \circ R^{23}=R^{23} \circ R^{13} \circ R^{12}=R^{12} \circ R^{23} \circ R^{12}$ $R^{23} \circ R^{13} \circ R^{12}=R^{23} \circ R^{12} \circ R^{23}=R^{12} \circ R^{13} \circ R^{23}$

The other claims follow in a similar manner. $\diamond$

We will write the above results in the following manner:

$$
(7) \wedge(8) \rightarrow(2) \wedge(3) ; \quad(2) \wedge(3) \wedge(12) \rightarrow(9) ;
$$

$(2) \vee(3) \rightarrow(10) ; \quad(12) \wedge[(2) \vee(3)] \rightarrow(11) . \quad$ Also, $\quad(10) \wedge(12) \rightarrow(13)$

As a direct application of this theorem, one can check which of the funtions presented in this section are common solutions for the braid condition and the quantum Yang-Baxter equation. 
Remark 3.10. The set of equations of operators of type $R: V \otimes V \rightarrow V \otimes V$ and $R^{i j}: V \otimes V \otimes V \rightarrow V \otimes V \otimes V$, with $i, j \in\{1,2,3\}$, has a natural distributive latice structure (see [25]).

\section{YANG-BAXTER SYSTEMS}

We present the Yang-Baxter systems theory following the paper [6]. Yang-Baxter systems were introduced in [11] as a spectral-parameter independent generalisation of quantum Yang-Baxter equations related to non-ultralocal integrable systems.

Yang-Baxter systems are conveniently defined in terms of Yang-Baxter commutators. Consider three vector spaces $V, V^{\prime}, V^{\prime \prime}$ and three linear maps $R: V \otimes V^{\prime} \rightarrow V \otimes V^{\prime}$, $S: V \otimes V^{\prime \prime} \rightarrow V \otimes V^{\prime \prime}$ and $T: V^{\prime} \otimes V^{\prime \prime} \rightarrow V^{\prime} \otimes V^{\prime \prime}$.

Then a Yang-Baxter commutator is a map $[R, S, T]: V \otimes V^{\prime} \otimes V^{\prime \prime} \rightarrow V \otimes V^{\prime} \otimes V^{\prime \prime}$, defined by

$$
[R, S, T]=R_{12} \circ S_{13} \circ T_{23}-T_{23} \circ S_{13} \circ R_{12} .
$$

In terms of a Yang-Baxter commutator, the quantum Yang-Baxter equation (3) is expressed simply as $[R, R, R]=0$.

Definition 4.1. Let $V$ and $V^{\prime}$ be vector spaces. A system of linear maps

$$
W: V \otimes V \rightarrow V \otimes V, \quad Z: V^{\prime} \otimes V^{\prime} \rightarrow V^{\prime} \otimes V^{\prime}, \quad X: V \otimes V^{\prime} \rightarrow V \otimes V^{\prime}
$$

is called a WXZ-system or a Yang-Baxter system, provided the following equations are satisified:

$$
\begin{gathered}
{[W, W, W]=0,} \\
{[Z, Z, Z]=0,} \\
{[W, X, X]=0,} \\
{[X, X, Z]=0 .}
\end{gathered}
$$

There are several algebraic origins and applications of WXZ-systems. WXZ-systems with invertible $W, X$ and $Z$ can be used to construct dually-paired bialgebras of the FRT type, thus leading to quantum doubles.

More precisely, consider a WXZ-system with finite-dimensional $V=V^{\prime}$, so that each of $W, X, Z$ is an $N^{2} \times N^{2}$-matrix. Suppose that $W, X, Z$ are invertible. Since $W$ and $Z$ satisfy Yang-Baxter equations (15)-(16), one can consider two matrix bialgebras $A$ and $B$ with $N \times N$ matrices of generators $U$ and $T$ respectively, and relations $W_{12} U_{1} U_{2}=U_{2} U_{1} W_{12}, Z_{12} T_{1} T_{2}=T_{2} T_{1} Z_{12}$. The existence of an invertible operator $X$ that satisfies equations (17)-(18), means that $A$ and $B$ are dually paired with a non-degenerate pairing $\left\langle U_{1}, T_{2}\right\rangle=X_{12}$. Furthermore, the tensor product $A \otimes B$ has an algebra (quantum double) structure with crossed relations $X_{12} U_{1} T_{2}=T_{2} U_{1} X_{12}$.

Given a WXZ-system as in Definition 4.1 one can construct a Yang-Baxter operator on $V \oplus V^{\prime}$, provided the map $X$ is invertible. This is a special case of a gluing procedure described in [23, Theorem 2.7] (cf. [23, Example 2.11]). Let $R=W \circ \tau_{V, V}$, $R^{\prime}=Z \circ \tau_{V^{\prime}, V^{\prime}}, U=X \circ \tau_{V^{\prime}, V}$. Then the linear map

$$
R \oplus_{U} R^{\prime}:\left(V \oplus V^{\prime}\right) \otimes\left(V \oplus V^{\prime}\right) \rightarrow\left(V \oplus V^{\prime}\right) \otimes\left(V \oplus V^{\prime}\right)
$$


given by $\left.R \oplus_{U} R^{\prime}\right|_{V \otimes V}=R,\left.R \oplus_{U} R^{\prime}\right|_{V^{\prime} \otimes V^{\prime}}=R^{\prime}$, and for all $x \in V, y \in V^{\prime}$,

$$
\left(R \oplus_{U} R^{\prime}\right)(y \otimes x)=U(y \otimes x), \quad\left(R \oplus_{U} R^{\prime}\right)(x \otimes y)=U^{-1}(x \otimes y)
$$

is a Yang-Baxter operator.

Entwining structures were introduced in order to recapture the symmetry structure of non-commutative (coalgebra) principal bundles or coalgebra-Galois extensions.

Definition 4.2. An algebra $A$ is said to be entwined with a coalgebra $C$ if there exists a linear map $\psi: C \otimes A \rightarrow A \otimes C$ satisfying the following four conditions:

(1) $\psi \circ\left(I_{C} \otimes \mu\right)=\left(\mu \otimes I_{C}\right) \circ\left(I_{A} \otimes \psi\right) \circ\left(\psi \otimes I_{A}\right)$,

(2) $\left(I_{A} \otimes \Delta\right) \circ \psi=\left(\psi \otimes I_{C}\right) \circ\left(I_{C} \otimes \psi\right) \circ\left(\Delta \otimes I_{A}\right)$,

(3) $\psi \circ\left(I_{C} \otimes \iota\right)=\iota \otimes I_{C}$,

(4) $\left(I_{A} \otimes \varepsilon\right) \circ \psi=\varepsilon \otimes I_{A}$.

The map $\psi$ is known as an entwining map, and the triple $(A, C)_{\psi}$ is called an entwining structure.

Remark 4.3. To denote the action of an entwining map $\psi$ on elements it is convenient to use the following $\alpha$-notation, for all $a, b \in A$ and $c \in C$,

$\psi(c \otimes a)=\sum_{\alpha} a_{\alpha} \otimes c^{\alpha}, \quad\left(I_{A} \otimes \psi\right) \circ\left(\psi \otimes I_{A}\right)(c \otimes a \otimes b)=\sum_{\alpha, \beta} a_{\alpha} \otimes b_{\beta} \otimes c^{\alpha \beta}$, etc.

For example (6) is a kind of $\alpha$-notation related to the Yang-Baxter equation.

The relations (1), (2), (3) and (4) in Definition 4.2 are equivalent to the following explicit relations, for all $a, b \in A, c \in C$,

$$
\begin{gathered}
\sum_{\alpha}(a b)_{\alpha} \otimes c^{\alpha}=\sum_{\alpha, \beta} a_{\alpha} b_{\beta} \otimes c^{\alpha \beta}, \\
\sum_{\alpha} a_{\alpha} \otimes c^{\alpha}{ }_{(1)} \otimes c^{\alpha}{ }_{(2)}=\sum_{\alpha, \beta} a_{\beta \alpha} \otimes c_{(1)}{ }^{\alpha} \otimes c_{(2)}{ }^{\beta}, \\
\sum_{\alpha} 1_{\alpha} \otimes c^{\alpha}=1 \otimes c, \\
\sum_{\alpha} a_{\alpha} \varepsilon\left(c^{\alpha}\right)=a \varepsilon(c) .
\end{gathered}
$$

Theorem 4.4. ([6]) Let $A$ be an algebra and let $C$ be a coalgebra. For any $s, r, t, p \in k$ define linear maps

$$
W: A \otimes A \rightarrow A \otimes A, \quad a \otimes b \mapsto s b a \otimes 1+r 1 \otimes b a-s b \otimes a,
$$

$Z: C \otimes C \rightarrow C \otimes C, \quad c \otimes d \mapsto t \varepsilon(c) \sum d_{(1)} \otimes d_{(2)}+p \varepsilon(d) \sum c_{(1)} \otimes c_{(2)}-p d \otimes c$.

Let $X: A \otimes C \rightarrow A \otimes C$ be a linear map such that $X \circ\left(\iota \otimes I d_{C}\right)=\iota \otimes I d_{C}$ and $\left(I d_{A} \otimes \varepsilon\right) \circ X=I d_{A} \otimes \varepsilon$. Then $W, X, Z$ is a Yang-Baxter system if and only if $A$ is entwined with $C$ by the map $\psi:=X \circ \tau_{C, A}$. 


\section{JORDAN ALGEBRAS AND UJLA STRUCTURES}

Jordan algebras have applications in physics, differential geometry, ring geometries, quantum groups, analysis, biology, etc (see [12, 14, 13]).

We have introduced some structures which unify Jordan algebras, Lie algebras and (non-unital) associative algebras. These structures were called UJLA (from "unification", "Jordan", "Lie" and "associative") structures, and one could "decode" the results obtained for UJLA structures in results for Jordan algebras, Lie algebras or (non-unital) associative algebras.

Changhing the perspective, one can consider the UJLA structures as generalizations of Jordan algebras.

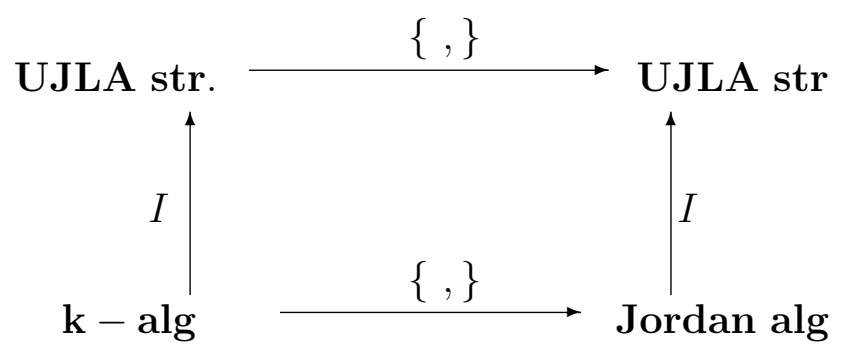

UJLA structures can also be interpretated as in an intermediat step in the process of associationg a Lie algebra to an associative algebra (see the picture below).

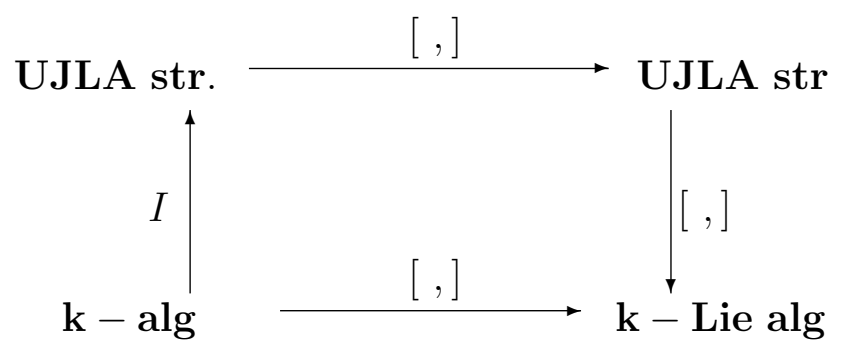

The study of filiform Lie algebras ([40]) can be extended to filiform UJLA structures.

Definition 5.1. We have defined the unifying structure $(V, \eta)$, also called a "UJLA structure", in the following way. Let $V$ be a vector space, and $\eta: V \otimes V \rightarrow V, \quad \eta(a \otimes$ $b)=a b$, be a linear map, which satisfies the following axioms $\forall a, b, c \in V$ :

$$
\begin{aligned}
(a b) c+(b c) a+(c a) b & =a(b c)+b(c a)+c(a b) \\
\left(a^{2} b\right) a & =a^{2}(b a) \\
(a b) a^{2} & =a\left(b a^{2}\right) \\
\left(b a^{2}\right) a & =(b a) a^{2} \\
a^{2}(a b) & =a\left(a^{2} b\right) .
\end{aligned}
$$

If just the identity (23) holds, we call the structure $(V, \eta)$ a "weak unifying structure". 
Remark 5.2. If $(A, \theta)$, where $\theta: A \otimes A \rightarrow A, \theta(a \otimes b)=a b$, is a (non-unital) associative algebra, then we define a UJLA structure $\left(A, \theta^{\prime}\right)$, where $\theta^{\prime}(a \otimes b)=\alpha a b+$ $\beta b a$, for some $\alpha, \beta \in k$. For $\alpha=\frac{1}{2}$ and $\beta=\frac{1}{2}$, then $\left(A, \theta^{\prime}\right)$ is a Jordan algebra, and for $\alpha=1$ and $\beta=-1$, then $\left(A, \theta^{\prime}\right)$ is a Lie algebra.

Theorem 5.3. ([32]) Let $(V, \eta)$ be a UJLA structure, and $\alpha, \beta \in k$. Then, $\left(V, \eta^{\prime}\right), \eta^{\prime}(a \otimes$ $b)=\alpha a b+\beta b a$ is a UJLA structure.

OPEN PROBLEM. A UJLA structure is power associative. We know that a UJLA structure is power associative for dimensions less or equal to 5.

Remark 5.4. The classification of UJLA structures is also an open problem.

Theorem 5.5. ([32]) Let $V$ be a vector space over the field $k$, and $p, q \in k$. For $f, g: V \rightarrow V$, we define $M(f \otimes g)=f * g=f *_{p, q} g=p f \circ g+q g \circ f: V \rightarrow V$. Then:

(i) $\left.\operatorname{End}_{k}(V), *_{p, q}\right)$ is a UJLA structure $\forall p, q \in k$.

(ii) For $\phi: \operatorname{End}_{k}(V) \rightarrow \operatorname{End}_{k}(V \otimes V)$ a morphism of UJLA structures (i.e., $\phi(f *$ $g)=\phi(f) * \phi(g)), W=\{f: V \rightarrow V \mid f \circ M=M \circ \phi(f)\}$ is a sub-UJLA structure of the structure defined at (i). In other words, $f * g \in W, \forall f, g \in W$.

Theorem 5.6. ([33]) Let $(V, \eta)$ be a UJLA structure. Then, $\left(V, \eta^{\prime}\right), \eta^{\prime}(a \otimes b)=a b-b a$ is a Lie algebra.

Proof. The proof follows from formula (23).

Theorem 5.7. ([33]) Let $(V, \eta)$ be a UJLA structure. Then, $\left(V, \eta^{\prime}\right), \quad \eta^{\prime}(a \otimes b)=$ $\frac{1}{2}(a b+b a)$ is a Jordan algebra.

Proof. The proof follows from formulas (24), (25), (26) and (27).

Theorem 5.8. ([31]) For $(V, \eta)$ a UJLA structure, $D(x)=D_{b}(x)=b x-x b$ is $a$ UJLA-derivative (i.e., $\left.D\left(a^{2} a\right)=D\left(a^{2}\right) a+a^{2} D(a) \forall a \in V\right)$.

Definition 5.9. For the vector space $V$, let $d: V \rightarrow V$ and $\phi: V \otimes V \rightarrow V \otimes V$, be a linear map which satisfies:

$$
\phi^{12} \circ \phi^{23} \circ \phi^{12}=\phi^{23} \circ \phi^{12} \circ \phi^{23}
$$

where $\phi^{12}=\phi \otimes I, \phi^{23}=I \otimes \phi, \quad I: V \rightarrow V, \quad a \mapsto a$.

Then, $(V, d, \phi)$ is called a generalized derivative if $\phi \circ(d \otimes I+I \otimes d)=(d \otimes I+I \otimes d) \circ \phi$.

Remark 5.10. If $A$ is an associtive algebra, $d: A \rightarrow A$ a derivative (so, $\left.d\left(1_{A}\right)=0\right)$, and $\phi: A \otimes A \rightarrow A \otimes A, a \otimes b \mapsto a b \otimes 1+1 \otimes a b-a \otimes b$, then $(A, d, \phi)$ is a generalized derivative.

If $C$ is a coalgebra, $d: C \rightarrow C$ a coderivative, and $\psi: C \otimes C \rightarrow C \otimes C, c \otimes d \mapsto$ $\varepsilon(d) c_{1} \otimes c_{2}+\varepsilon(c) d_{1} \otimes d_{2}-c \otimes d$, then $(C, d, \psi)$ is a generalized derivation.

If $\tau$ is the twist map, the condition

$$
\tau \circ R \circ \tau=R
$$

represents the unification of the comutativity and the co-comutativity conditions. In other words, if the algebra $A$ is comutative, then $\phi$ verifies the above condition. If the coalgebra $C$ is cocomutative, then $\psi$ verifies the same condition. 
Definition 5.11. Let $A$ is an associtive algebra, $d: A \rightarrow A$ a derivative, $M$ an A-bimodule, and $D: M \rightarrow M$ with the property $D(a m)=d(a) m+a D(m)$. Then, $(A, d, M, D)$ is called a module derivative.

Theorem 5.12. ([30]) In the above case, the following are true:

(i) $A \times M$ becomes an algebra;

(ii) $\delta: A \times M \rightarrow A \times M,(a, m) \mapsto(d(a), D(m))$ is a derivative in this algebra.

Translated into the "language" of Differential Geometry, the above theorem says that the Lie derivative is a derivative (i.e., $d(a b)=d(a) b+a d(b))$ on the product of the algebra of functions defined on the manifold $M$ with the set of vector fields on $M$ (see [22]).

Remark 5.13. A dual construction would refer to a coalgebra structure, $\Delta: A \rightarrow$ $A \otimes A, f \mapsto f \otimes 1+1 \otimes f$, and a comodule structure on forms, $\rho: \Omega \rightarrow A \otimes \Omega, f d x_{1} \wedge$ $d x_{2} \ldots \wedge d x_{n} \mapsto f \otimes d x_{1} \wedge d x_{2} \ldots \wedge d x_{n}+1 \otimes f d x_{1} \wedge d x_{2} \ldots \wedge d x_{n}$.

$A \times \Omega$ becomes a coalgebra with the following comultiplication:

$\left(f, g d x_{1} \wedge d x_{2} \ldots \wedge d x_{n}\right)=(f, 0)+(0, g \omega) \mapsto(f, 0) \otimes(1,0)+(1,0) \otimes(f, 0)+(g, 0) \otimes$ $(0, \omega)+(1,0) \otimes(0, g \omega)+(0, \omega) \otimes(g, 0)+(0, g \omega) \otimes(1,0)$.

We can see now that the Lie derivative is a coderivative with the above comultiplication, $\Delta$. The key ingredient in the above remark is a theorem given below.

Theorem 5.14. ([31]) Let $C$ be an associative algebra and $M$ a $C$-bicomodule. Then, $C \times M$ becomes a coalgebra.

Proof. One has to define a comultiplication on $C \times M$,

$\Delta_{C \times M}(c, 0)=\sum\left(c_{1}, 0\right) \otimes\left(c_{1}, 0\right), \Delta_{C \times M}(0, m)=\sum\left(m_{-1}, 0\right) \otimes\left(0, m_{0}\right)+\sum\left(0, m_{0}\right) \otimes$ $\left(m_{1}, 0\right)$, and a counity $\varepsilon_{C \times M}(c, m)=\varepsilon_{C}(c)$.

The axioms of coalgrebras are easily verified.

Remark 5.15. The unification of Theorem 5.12 and the construction from Remark 5.13 can be realized at the level of Yang-Baxter systems.

For example, $W(f \otimes g)=1 \otimes f g, X(f \otimes v)=1 \otimes f v$ and $Z(v \otimes w)=0$ forms $a$ Yang-Baxter systems.

Dually, $W(f \otimes g)=\varepsilon(f) \otimes \Delta(g), X(f \otimes v)=\varepsilon(f) \otimes \rho(v)$ and $Z(v \otimes w)=0$ forms a Yang-Baxter systems.

We have explained that gluing procedures for Yang-Baxter systems lead to YangBaxter operators. The algebraic part of this unification is presented below. More details and interpretations will follows in later studies.

Theorem 5.16. Let $V$ and $V^{\prime}$ be vector spaces, and

$$
W: V \otimes V \rightarrow V \otimes V, \quad X: V \otimes V^{\prime} \rightarrow V \otimes V^{\prime}, \quad Y: V^{\prime} \otimes V \rightarrow V^{\prime} \otimes V
$$

such that the following equations are satisified:

$$
\begin{gathered}
{[W, W, W]=0,} \\
{[W, X, X]=0,} \\
{[Y, Y, W]=0,} \\
{[X, W, Y]=0 .}
\end{gathered}
$$


Then the linear map $\quad R:\left(V \oplus V^{\prime}\right) \otimes\left(V \oplus V^{\prime}\right) \rightarrow\left(V \oplus V^{\prime}\right) \otimes\left(V \oplus V^{\prime}\right)$, given by $\left.R\right|_{V \otimes V}=W,\left.\quad R\right|_{V \otimes V^{\prime}}=X,\left.\quad R\right|_{V^{\prime} \otimes V}=Y$, and $\left.R\right|_{V^{\prime} \otimes V^{\prime}}=0$, has the property $[R, R, R]=0$.

Proof. The proof is based on observations on the decomposition of $\left(V \oplus V^{\prime}\right)^{\otimes 3}$ into direct summands of tensor products.

Remark 5.17. Let $A$ is an associtive algebra, $W: A \otimes A \rightarrow A \otimes A, a \otimes b \mapsto$ $a b \otimes 1+1 \otimes a b-b \otimes a, M$ an A-bimodule, $X: A \otimes M \rightarrow A \otimes M, a \otimes m \mapsto 1 \otimes a m$, and $Y: M \otimes A \rightarrow M \otimes A, n \otimes b \mapsto n b \otimes 1$, then we have the conditions of the above theorem fulfilled.

\section{Conclusions}

We proposed an open problem which generalities Tzitzeica-Johnson's theorem: the circles from that theorem are replaced by an arbitrary closed convex curve, and the conclusion is slightly weakened.

The story of the number $\pi$ was then continued with a new episode.

A review (and new results) on Yang-Baxter systems/equations were needed for unifying two constructions. Theorem 5.16 unifyies Theorem 5.12 (i) and Theorem 5.14 .

The Vrănceanu - Vergne basis was mentioned in a talk at DGA 13, which later became a paper $([40])$.

\section{ACKNOWLEDGMENT}

We would like to thank Professors Radu Iordănescu, Lazlo Stacho and Nicolae Anghel for their help and suggestions. Also, we thank the Simion Stoilow Institute of Mathematics of the Romanian Academy and the Petroleum Gas University from Ploiesti.

\section{REFERENCES}

[1] N. Anghel, Heron Triangles with constant area and perimeter, talk, Differential Geometry and its Applications, UPG, 9 - 11 July, 2019, submitted to Revue Roumaine de Mathematiques Pures et Appliquees.

[2] R.J. Baxter, Exactly Solved Models in Statistical Mechanics, Acad. Press, London,1982.

[3] R.J. Baxter, Partition function for the eight-vertex lattice model, Ann. Physics 70(1972), 193-228.

[4] Paolo Bellingeri, Virtual braid groups and permutations, talk, Differential Geometry and its Applications, UPG, 9 - 11 July, 2019.

[5] Tomasz Brzeziński, Towards semi-trusses Revue Roumaine de Mathematiques Pures et Appliquees, No. 2 (2018), pp. 75-89.

[6] T. Brzezinski, T.; Nichita, F.F. Yang-Baxter systems and entwined structures, Communications in Algebra 2005, vol. 33(4), pp. 1083-1093.

[7] F. Caragiu, A. Lemeni, S. Trausan-Matu, Language in Mathematics, Science and Artificial Intelligence vs. Language in Theology, Dialog in Absolut / ieromonah Ghelasie Gheorghe, Bucuresti: Platytera, 2007, 293-3007.

[8] S. Dăscălescu and F. F. Nichita, Yang-Baxter operators arising from (co)algebra structures. Comm. Algebra 1999, 27, 5833-5845.

[9] P. Freyd, D. Yetter, J. Hoste, W. B. R. Lickorish, K. Millett, A. Ocneanu, A new polynomial invariant of knots and links, Bull. Amer. Math. Soc. 12 (1985), 239-246.

[10] P. Greco, Povestea numarului PI, HUMANITAS, 2019 (translated by Liviu Ornea). 
[11] L. Hlavaty and L. Snobl, Solution of the Yang-Baxter system for quantum doubles, Int. J. Mod. Phys. A14 (1999), 3029-3058.

[12] R. Iordanescu, Jordan structures in mathematics and physics. Mathematics 2011, http://arxiv.org/abs/1106.4415.

[13] R. Iordanescu, Romanian contributions to the study of Jordan structures and their applications, Mitteilungen des Humboldt-Clubs Rumanien 2004-2005, No. 8-9, Bukarest, 29-35.

[14] R. Iordanescu, Jordan structures in geometry and physics with an Appendix on Jordan structures in analysis, Romanian Academy Press, 2003.

[15] R. Iordanescu, F.F. Nichita and I.M. Nichita, The Yang-Baxter Equation, (Quantum) Computers and Unifying Theories. Axioms 2014, 3, 360-368.

[16] R. Iordanescu, F.F. Nichita and I.M. Nichita, Non-associative algebras, Yang-Baxter equations and quantum computers. Bulgarian Journal of Physics 2014, vol.41 n.2, 71-76.

[17] M. Jimbo, Quantum $R$ matrix for the generalized Toda system, Lett. Math. Phys. 11 (1986), 247-252.

[18] V. F. R. Jones, A polynomial invariant for knots via von Neumann algebras, Bull. Amer. Math. Soc. 12 (1985), 103-111.

[19] L. H. Kauffman, New invariants in the theory of knots, Astérisque 163-164 (1988), 137-219.

[20] V. Lebed, Braided Systems: A Unified Treatment of Algebraic Structures with Several Operations, Homology, Homotopy and Applications, vol. 19(2), (2017), pp. 141-174.

[21] V. Lebed, Homologies of algebraic structures via braidings and quantum shuffles, Journal of Algebra 391, 152-192 (2013).

[22] Lie derivative, From Wikipedia, the free encyclopedia, https://en.wikipedia.org/wiki/Lie_derivative

[23] Majid, S., Markl, M., Glueing operations for R-Matrices, quantum groups and link-invariants of Hecke type, Math. Proc. Cambridge Philos. Soc. 119 (1996), 139-166.

[24] G. Massuyeau and F.F. Nichita, Yang-Baxter operators arising from algebra structures and the Alexander polynomial of knots, Comm. Algebra 2005, 33 (7) 2375-2385.

[25] Mocanu, C.; Nichita, F.F.; Pasarescu, O. Applications of Non-Standard Analysis in Topoi to Mathematical Neuroscience and Artificial Intelligence: I. Mathematical Neuroscience. Preprints 2020, 2020010102 (doi: 10.20944/preprints202001.0102.v1).

[26] Moise, L.D.; Cristea, R Gheorghe Titeica - reverbertii si permanente ale geometriei in contemporaneitate, Proceedings, Conferinta Nationala de Invatamant Virtual, editia a XI-a, 2013, $150-156$.

[27] J. Murakami, A state model for the multi-variable Alexander polynomial, Pacific J. Math. 157, 1 (1993), 109-135.

[28] Florin Nichita, On Applications of UJLA Structures, talk, Differential Geometry and its Applications, UPG, 9 - 11 July, 2019.

[29] Nichita, F.F. Unification Theories: Examples and Applications. Axioms 2018, 7, 85.

[30] Nichita, F.F. Unification Theories: New Results and Examples. Axioms 2019, 8, 60.

[31] Nichita, F.F. Mathematics and Poetry • Unification, Unity, Union. Sci 2020, 2, 58.

[32] Nichita, F.F., Yang-Baxter Equations, Computational Methods and Applications. Axioms 2015, 4, 423-435.

[33] Nichita, F.F., On Jordan algebras and unification theories, Revue Roumaine de Mathematiques Pures et Appliquees 61(4), 2016, 305-316.

[34] Nichita, F.F. (Ed.) Hopf Algebras, Quantum Groups and Yang-Baxter Equations, MDPI, 2019, ISBN 978-3-03897-324-9 (Pbk); ISBN 978-3-03897-325-6 (PDF).

[35] F.F. Nichita, Self-Inverse Yang-Baxter Operators from (Co)Algebra structures, Journal of Algebra, Volume 218, Number 2, 738-759(1999).

[36] J. H.H. Perk and H. Au-Yang, Yang-Baxter Equations, Encyclopedia of Mathematical Physics, eds. J.-P. Françoise, G.L. Naber and Tsou S.T., Oxford: Elsevier, 2006 (ISBN 978-0-1251-2666-3), volume 5, pages 465-473.

[37] https://it.wikipedia.org/wiki/Pi_greco ; https://it.wikipedia.org/wiki/Pietro_Greco

[38] Radu Popescu, Resonance varieties. Definition and applications, talk, Differential Geometry and its Applications, UPG, 9 - 11 July, 2019.

[39] J. H. Przytycki, P. Traczyk, Invariants of links of Conway type, Kobe J. Math. 4, 2 (1987), 115-139. 
[40] Elisabeth Remm, On filiform Lie algebras. Geometric and algebraic studies, Revue Roumaine de Mathematiques Pures et Appliquees, Selected papers from the 13th International Workshop on Differential Geometry and its Applications (September, 2017) TOME LXIII, NO 2, 2018, 179-209.

[41] Elisabeth Remm, Rigidity of Lie algebras, poster, Differential Geometry and its Applications, UPG, 9 - 11 July, 2019, submitted to Revue Roumaine de Mathematiques Pures et Appliquees.

[42] Ibrahim SENTURK, Tahsin ONER, Tugce KALKAN and Mehmet TERZILER, A perspective on the Yang-Baxter equation in BCK algebras, preprint.

[43] V. Turaev, The Yang-Baxter equation and invariants of links, Invent. Math. 1988 92 527-553.

[44] C. N. Yang, Some exact results for the many-body problem in one dimension with repulsive delta-function interaction, Phys. Rev. Lett. 19(1967), 1312-1315. 